\title{
Historia de vida que empodera
}

\section{Life story that empowers}

TERRAZAS-MEDINA, Tamara Isabel†*, MARTÍNEZ-CÁRDENAS, Juana María, MORALESSILVA, Rosa Elia y PÉREZ-CASTRO, Francisco Isaí

Universidad Autónoma de Coahuila, Escuela de Bachilleres Dr. Mariano Narváez Glz. T.M.

ID $1^{\text {er }}$ Autor: Tamara Isabel, Terrazas-Medina / ORC ID: 0000-0002-6581-190X, arXiv Author ID: 1909.09062, CVU CONACYT ID: 929839

ID $1^{\text {er }}$ Coautor: Juana María, Martínez-Cárdenas / ORC ID: 0000-0003-1004-9652, Researcher ID Thomson: X-23702018, CVU CONACYT ID: 949979

ID $2^{\text {do }}$ Coautor: Rosa Elia, Morales-Silva / ORC ID: 0000-0001-6299, CVU CONACYT ID: 950197

ID $3^{\text {er }}$ Coautor: Francisco Isaí, Pérez-Castro / ORC ID: 0000-0002-6779-7881, CVU CONACYT ID: 929950

DOI: $10.35429 / J T M S .2019 .17 .5 .9 .15$

Recibido 30 de Julio 2019; Aceptado 30 Diciembre, 2019

\section{Resumen}

Esta investigación presenta los resultados de un estudio realizado con Historia de Vida, aquí se expone el proceso de empoderamiento de una niña de diez años, a través del desarrollo de su Inteligencia Emocional. El objetivo fue: Analizar el contexto escolar y familiar para obtener las variables de mayor influencia en el logro de su empoderamiento. Se señala la estrategia utilizada para lograrlo, lo cual involucró a la escuela y la familia. La pregunta de investigación se planteó así: ¿Cómo es el proceso de empoderamiento de una niña de diez años y qué es lo que influye en ella para lograrlo? La recolección de los datos fue mediante entrevistas semiestructuradas las cuales fueron trianguladas para dar validez a esta investigación. El aporte científico es la identificación y conocimiento de las dimensiones y variables que más influyen en el empoderamiento de la niña. Se concluye que dos de las variables más importantes para el logro de lo anterior, son las relativas a la dimensión de la competencia personal, lo que colabora fuertemente en el empoderamiento de esta estudiante de primaria.

\section{Empoderamiento infantil, Inteligencia Emocional, Estrategias de logro}

\begin{abstract}
This research presents the results of a study conducted with Life History, here is the process of empowering a ten-year-old girl, through the development of her Emotional Intelligence. The objective was to: Analyze the school and family context to obtain the variables with the greatest influence in achieving their empowerment. The strategy used to achieve this is indicated, which involved the school and the family. The research question was posed as follows: How is the process of empowering a ten-year-old girl and what influences her to achieve it? The data collection was through semi-structured interviews which were triangulated to validate this research. The scientific contribution is the identification and knowledge of the dimensions and variables that most influence the empowerment of the girl. It is concluded that two of the most important variables for the achievement of the above, are those related to the dimension of personal competence, which strongly collaborates in the empowerment of this elementary student.
\end{abstract}

Child empowerment, Emotional intelligence, Achievement strategies

Citación: TERRAZAS-MEDINA, Tamara Isabel, MARTÍNEZ-CÁRDENAS, Juana María, MORALES-SILVA, Rosa Elia y PÉREZ-CASTRO, Francisco Isaí. Historia de vida que empodera. Revista Transdisciplinaria de Estudios Migratorios. 2019, 5-17: 9-15

\footnotetext{
* Correspondencia al Autor (correo electrónico: tamy0725@ gmail.com)

$\dagger$ Investigador contribuyendo como primer Autor.
} 


\section{Introducción}

En la actualidad, diferentes organismos internacionales y nacionales, han trabajado en pro del bienestar de los niños, tratando algunos de ellos de "empoderarlos", con la finalidad de que su desempeño social, educativo, físico, valoral, etc., se vea desarrollado y que impacte su quehacer en cualquier contexto. La escuela y la familiar son espacios idóneos para colaborar en ese desarrollo pues son, por cercanía, los primeros promotores del avance de sus competencias.

A través del tiempo, en las culturas y en las diferentes etapas de la humanidad, se ha concebido en distintos modos las maneras de educar y formar a los infantes, incluso la conceptualización de la infancia, ha sido distinta. Las leyes en todos los tiempos, han entendido diferente la idea sobre niñez, así tenemos, por ejemplo, en Grecia y Roma antiguas, la niñez no estaba ligada al concepto de persona (Álvarez, 2011, p. 1). Ya en la Grecia clásica se abogaba por que los hombres se escolarizaran en lo informal (referente a la lectura, escritura y la cuestión física), luego, hacia lo formal: literatura, aritmética, filosofía y ciencia.

Ileana Enesco (s/f), menciona distintas fases históricas del concepto de infancia:

- $\quad$ Cristianismo y Edad Media. La iglesia controlaba la educación, se educaba para servir a Dios. El niño era concebido como un ser perverso que debía ser socializado y reformado. En este tiempo el niño era utilizado como mano de obra. Del Renacimiento al S. XVII. En el Renacimiento, se produce interés por el desarrollo infantil, pero se le considera como homúnculo. Con la Revolución Industrial, los niños ya no fueron necesarios para producir, por lo que la escolarización se convirtió en el objetivo principal.

Siglos XVIII y XIX. En Estados Unidos e Inglaterra se decía que el niño debía ser reformado mediante el castigo físico. En Europa se abogaba por la adaptación de la educación al nivel del niño, se defendían las ideas sobre su bondad y, la obligatoriedad de la educación (incluso para las mujeres). Aún no había una concepción unificada de la infancia y la educación.
Las ideas anteriores, presentan las concepciones de infancia y educación que las distintas épocas históricas y comunidades han tenido sobre el tema. En la actualidad mexicana, "la carta magna les reconoce a la infancia y la adolescencia los derechos humanos y garantías individuales que consagra para todas las personas, destacando entre éstos, los contemplados en los artículos $1^{\circ}$., $2^{\circ}$., $3^{\circ}$., $4^{\circ}$., 18 y 123" (Cárdenas, S/F, p. 41).

Para la UNICEF (2005), en México y el mundo, la infancia es una etapa que se debe de proteger a toda costa y garantizar los derechos fundamentales:

"La infancia es la época en la que los niños y niñas tienen que estar en la escuela y en los lugares de recreo, crecer fuertes y seguros de sí mismos y recibir el amor y el estímulo de sus familias y de una comunidad amplia de adultos. Es una época valiosa en la que los niños y las niñas deben vivir sin miedo, seguros frente a la violencia, protegidos contra los malos tratos y la explotación. Como tal, la infancia significa mucho más que el tiempo que transcurre entre el nacimiento y la edad adulta. Se refiere al estado y la condición de la vida de un niño, a la calidad de esos años". (UNICEF, 2005)

Un aspecto de suma relevancia indicado por la UNICEF es cuando dice: "crecer fuertes y seguros de sí mismos" (UNICEF, 2005), lo anterior no sólo depende de la presencia positiva de adultos y entornos favorables, depende también de la pertinencia con que la personalidad del niño se vaya formado $y$ desarrollando. En este sentido, la UNICEF menciona que una de las vías para "crecer fuertes y seguros de sí mismos" (UNICEF, 2005), es "el empoderamiento, o el proceso de fortalecer conocimientos y capacidades para ejercer el poder y tener la libertad, información y apoyo para tomar decisiones y actuar por hacerlas realidad" (UNICEF, 2017).

La conceptualización anterior lleva a entender que la infancia está siendo revalorada y, prevé en los niños capacidades para desarrollar competencias y usarlas en su favor y de otros. No obstante, lograr evidenciar lo anterior no es sencillo, es un trabajo arduo pues integra actores múltiples como la familia, los docentes, el entorno, la institución educativa y la misma currícula. 
Una de las vías para promover el empoderamiento infantil es la práctica de la Inteligencia Emocional, pues representa un entablado educativo holístico (dentro y fuera de la escuela) que orienta a la niñez hacia diferentes tipos de empoderamiento.

La Inteligencia Emocional (IE) "es el uso inteligente de las emociones: hacer que, intencionalmente, las emociones trabajen para nosotros, utilizándolas de manera que nos ayuden a guiar la conducta y los procesos de pensamiento, a fin de alcanzar el bienestar personal (Vivas, et al. 2007)".

Lo anterior lleva a reflexionar en la importancia que los procesos interpersonales e intrapersonal tienen $\mathrm{y}$, cómo impactan en el desarrollo de una persona. En este sentido es importante que el concepto actual de niñez, se desarrolle bajo una visión que promueva su propio crecimiento visionando puertas de futuro.

Autores como Goleman (1995), Vivas (2007) y Extremera y Fernández-Berrocal (2004), en entre otros, coinciden en que la Inteligencia Emocional (concepto que sigue en evolución) es la aptitud para sentir, entender, controlar y modificar emociones en uno mismo e influir en otros, con la intención de que las relaciones interpersonales sean cada vez mejores.

El mundo actual y sus requerimientos solicitan personas "competentes" en todos los sentidos, se piensa en seres que resuelven con efectividad problemas, que se integran a comunidades de aprendizaje distantes, en personas que colaboran y trabajan en pro de los demás, etc., y lo anterior se logra con el moldeo integral de la persona. Goleman ha documentado investigaciones (1998) donde se demuestra que el éxito del individuo está en el ser pertinente dentro del contexto en donde se encuentra y no tanto del coeficiente intelectual de la persona

En una recopilación de evidencias empíricas sobre el tema y, observando de cerca los resultados con estudiantes, Extremera y Fernández-Berrocal (2004), encontraron “...que los alumnos emocionalmente inteligentes, como norma general, poseen mejores niveles de ajuste psicológico y bienestar emocional, presentan una mayor calidad y cantidad de redes interpersonales y de apoyo social, son menos propensos a realizar comportamientos disruptivos, agresivos o violentos.
Pueden llegar a obtener un mayor rendimiento escolar al enfrentarse a las situaciones de estrés con mayor facilidad y consumen menor cantidad de sustancias adictivas (p.e., tabaco, alcohol, etc.)". Así mismo Lantieri (2009) enfatiza en el hecho de que el éxito académico se ve influido en mucho, por las sanas relaciones sociales que benefician los estados de ánimo del estudiante.

Fernández (2008) llega a la conclusión de que la enseñanza de emociones inteligentes depende de la práctica, el entrenamiento y su perfeccionamiento $\mathrm{y}$, no tanto, de la instrucción verbal. De la misma forma, Fernández (2011) concluye en su estudio que

"para lograr un desarrollo integral de los alumnos es necesario promover el desarrollo en las diferentes vertientes: afectiva, cognitiva y social. La educación emocional debe estar presente en la escuela dentro del proceso de desarrollo educativo de todo nuestro alumnado, como un proceso continuo y permanente que persiga formar personas más libres. Comunicar sus necesidades con precisión, solicitar ayuda, manejar la ansiedad, tomar un papel activo y controlar su lenguaje o, incluso, acomodar la forma de relación y convivir con compañeros de acuerdo a unas normas, se convierten en habilidades básicas a desarrollar".

Es importante observar cómo el concepto de Inteligencia Emocional tiene relevancia en el sector educativo actual, pues según los anteriores investigadores la IE contiene elementos que de ser desarrollados entre los estudiantes inciden en el logro de sus propósitos, no sólo educativos sino también de vida.

De la forma anterior, Lantieri (2009, p., 37) menciona: "numerosos estudios han descubierto que los jóvenes que poseen y dominan las habilidades sociales y emocionales son más felices, tiene más confianza en sí mismos y son más competentes como estudiantes, miembros familiares, amigos y trabajadores". La misma autora señala (2009, p., 35) "pareciera que el CI aporta sólo el 20 por ciento de los factores determinantes del éxito en la vida". Teniendo en cuenta los anteriores planteamientos se define la siguiente pegunta de investigación: ¿Cómo es el proceso de empoderamiento de una niña de diez años y qué es lo que influye en ella para lograrlo?

TERRAZAS-MEDINA, Tamara Isabel, MARTÍNEZ-CÁRDENAS Juana María, MORALES-SILVA, Rosa Elia y PÉREZ-CASTRO, Francisco Isaí. Historia de vida que empodera. Revista Transdisciplinaria de Estudios Migratorios. 2019 
Se persigue el siguiente objetivo general:

Analizar el contexto escolar y familiar para obtener las variables de mayor influencia en el logro del empoderamiento. Guía de investigación: El desarrollo de la Inteligencia Emocional en los niños influye en el empoderamiento de los mismos.

\section{Desarrollo}

Goleman (1995) considera que la Inteligencia Emocional es la capacidad para reconocer, comprender y regular nuestras emociones y las de los demás. Desde esta perspectiva, la Inteligencia Emocional es una habilidad que implica tres procesos:

1. Percibir: reconocer de forma consciente nuestras emociones e identificar qué sentimos y ser capaces de darle una etiqueta verbal.

2. Comprender: integrar lo que sentimos dentro de nuestro pensamiento y saber considerar la complejidad de los cambios emocionales.

3. Regular: dirigir y manejar las emociones tanto positivas como negativas de forma eficaz.

En este sentido, en el siguiente esquema se presentan las tres primeras competencias que hacen referencia a la competencia personal y las dos últimas a la competencia social. La competencia personal y la competencia social conforman la Inteligencia Emocional.

\section{El marco de la competencia emocional}

\begin{tabular}{|c|}
\hline $\begin{array}{l}\text { Competencia Personal. } \\
\text { Estas competencias determinan el modo en que nos } \\
\text { relacionamos con nosotros mismos. }\end{array}$ \\
\hline $\begin{array}{l}\text { Conciencia de uno mismo } \\
\text { Conciencia de nuestros propios estados internos, recursos e } \\
\text { intuiciones. }\end{array}$ \\
\hline $\begin{array}{l}\text { Autorregulación. } \\
\text { Control de nuestros estados, impulsos y recursos internos. }\end{array}$ \\
\hline $\begin{array}{l}\text { Motivación. } \\
\text { Las tendencias emocionales que guían o facilitan el logro de } \\
\text { nuestros objetivos. }\end{array}$ \\
\hline $\begin{array}{l}\text { Competencia Social. } \\
\text { Estas competencias determinan el modo en que nos } \\
\text { relacionamos con los demás. }\end{array}$ \\
\hline $\begin{array}{l}\text { Empatía. } \\
\text { Conciencia de los sentimientos, necesidades } \\
\text { preocupaciones ajenas. }\end{array}$ \\
\hline $\begin{array}{l}\text { Habilidades sociales. } \\
\text { Capacidad para inducir respuestas deseables en los demás. }\end{array}$ \\
\hline
\end{tabular}

Taba 1 El Marco de la Competencia Emocional. Fuente: La práctica de la inteligencia emocional, Daniel Goleman (1999)
Lo anterior planteado muestra la forma teórica en que cada persona puede desarrollarse de manera efectiva, la práctica de esta da la pauta para el logro de objetivos concretos, pues se trabaja con el interior y el exterior de la persona (competencias: personal y social). La revisión de la literatura ha mostrado la eficacia de su implementación en momentos educativos.

\section{Metodología}

Esta investigación está desarrollada con base en Historias de Vida, misma que busca entender las interacciones y acuerdos tácitos de la vida y realidad de esta niña empoderada. Historias de Vida fue elegido por ser un "método de investigación potente para conocer cómo las personas y el mundo que rodean al individuo influyen" (Hernández, 2009). La Historia de Vida que así se analiza, corresponde al denominado caso único, donde la seleccionada es una niña de diez años (que aquí llamaremos BILT). La selección obedece a las siguientes características: fácil oralidad, destaca en su comunidad por su labor científica, artística, social y política.

\section{Resultados}

Los resultados obtenidos apuntan a que el empoderamiento infantil está relacionado y respaldado por el desarrollo continuo de la Inteligencia Emocional. BILT cursa el 5to año de primaria, sus calificaciones son buenas, pero destaca más por su liderazgo y sensibilidad hacia los otros. Es tolerante y conciliadora, pero no siempre fue así. Su guía y familia trabajaron con ella en un tiempo, pues no entregaba tareas y presentaba un cuadro de pasividad poco comunes.

Se estableció una estrategia para el desarrollo de la inteligencia emocional de BILT, que incluía el trabajo en las áreas personales y sociales, tanto en casa como en la escuela.

La estrategia inició con el trabajo para desarrollar de la competencia personal fomentada en el entorno escolar y familiar, con este plan de trabajo BILT percibió que su guía trataba de motivarla utilizando diferentes materiales de aprendizaje, la niña comenta que no entendía su desgano, finalmente lo atribuyó al aburrimiento. 
Menciona que su guía trabajaba con ella y le mostraba diferentes materiales para una sola actividad educativa, esto la llevó a descubrir que podía elegir en forma consciente las cosas y por lo tanto poder resolverlas bajo distintas ópticas. El cambio en casa, BILT, lo relaciona con el cambio en la escuela y fue entonces que pudo reconocer que el aburrimiento es provocado por ella misma y, que sus emociones en relación a su trabajo áulico y en casa sólo dependen de ella.

Es importante mencionar que, a partir de este momento reconoció su conciencia emocional, lo que los autores del tema de Inteligencia Emocional entienden como una de las bases de la verdadera transformación, por otra parte, el empoderamiento de BILT, se asocia con la idea de "crecer fuertes y seguros de sí mismos” (UNICEF, 2005).

El segundo paso fue trabajar en la visión de sí misma, BILT ya conoce que sus acciones no sólo influyen en ella, también a los demás, así recuerda que es la presidenta del club de ciencia de su ambiente de aprendizaje y es la "cuidadora" del vivero de su escuela.

Para realizar esas actividades ha tendido que ser guía también de otros niños, esto la ha fortalecido, pues explicando aprende. Reconoce además que es muy buena haciendo manualidades y enseña a otros. Por otra parte, menciona que debe ser ejemplo para los otros niños pues ella es integrante del cabildo infantil de su localidad (ganó su lugar por concurso de oposición).

Sabe que lo anterior no sería posible sin la colaboración de su familia y su guía. Conoce sus fortalezas y ayuda a sus compañeros a conocer las de ellos, en este sentido les invita a hacer prácticas de diferentes cosas, ayudándoles a elegir lo que verdaderamente quieren y pueden hacer. En este sentido Goleman (1998, 32) menciona que "competencias emocionalescombinan el pensamiento y la emoción. Independientemente de los elementos cognitivos que intervengan, las competencias emocionales implican cierto grado de dominio de los sentimientos, una cualidad que contrasta agudamente con las aptitudes meramente cognitivas, que pueden realizar tanto una persona como un ordenador adecuadamente programado".
BILT, ha aprendido a conocerse bien y en este sentido, sabe las situaciones que le causan ciertas emociones, esto es importante pues se puede apartar a tiempo de aquello que no puede controlar o lo puede preparar.

Su guía la califica como íntegra, defensora de sus ideas, defensora de otros, etc., es una chica muy creativa y responsable, pues condujo a su equipo de ciencias a la etapa estatal de la Feria de las Ciencias y la Creatividad (COECYT-CONACYT), con la elaboración de un detergente biodegradable, que elimina el mal olor corporal. Esto la ha llevado a que sus acciones científicas sean reconocidas en el ámbito escolar. En este sentido se puede decir que BILT es una niña que está transitando al "empoderamiento, o el proceso de fortalecer conocimientos y capacidades" (UNICEF, 2017)

Desde que encontró que las cosas por hacer, sólo dependen de ella y de su actitud (paso 1 de la estrategia), sus acciones cambiaron (paso 2 de la estrategia), y a partir de aquí (situación derivativa de los pasos anteriores), los logros que ha obtenido responden a la alta motivación que desde ella nace por hacer las cosas, el grado de compromiso es alto de tal manera que alienta el trabajo de otros. Esto la ha llevado a establecer niveles de empatía elevados que fortalecen lazos con sus compañeros de aula.

BILT ha destacado ampliamente por sus habilidades sociales, es conciliadora, resuelve problemas con facilidad, sabe trabajar en equipo, es colaborativa y cooperativa. Los logros que BILT ha obtenido no son pocos, es la 8va, Regidora del Cabildo Infantil, "Cuidadora" del vivero escolar, Coordinadora del Club de Ciencias de su escuela, Líder del equipo de investigación científica de su escuela y miembro de una Asociación de escritores y poetas.

En las actividades anteriores ha destacado en la labor social, artística, científica y política. Y es justamente a partir de esas incursiones que el Cabildo de la Ciudad la ha elegido como imagen infantil de Saltillo, Coahuila. Su participación en esta ocasión está centrada en la promoción turística de la entidad. 
La Inteligencia Emocional "es el uso inteligente de las emociones: hacer que, intencionalmente, las emociones trabajen para nosotros, utilizándolas de manera que nos ayuden a guiar la conducta y los procesos de pensamiento, a fin de alcanzar el bienestar personal (Vivas, et al. 2007)", siguiendo esta definición, se puede decir que la estrategia seguida con BILT ha resultado exitosa y ha derivado en una niña empoderada: "el empoderamiento, o el proceso de fortalecer conocimientos y capacidades para ejercer el poder y tener la libertad, información y apoyo para tomar decisiones y actuar por hacerlas realidad" (UNICEF, 2017).
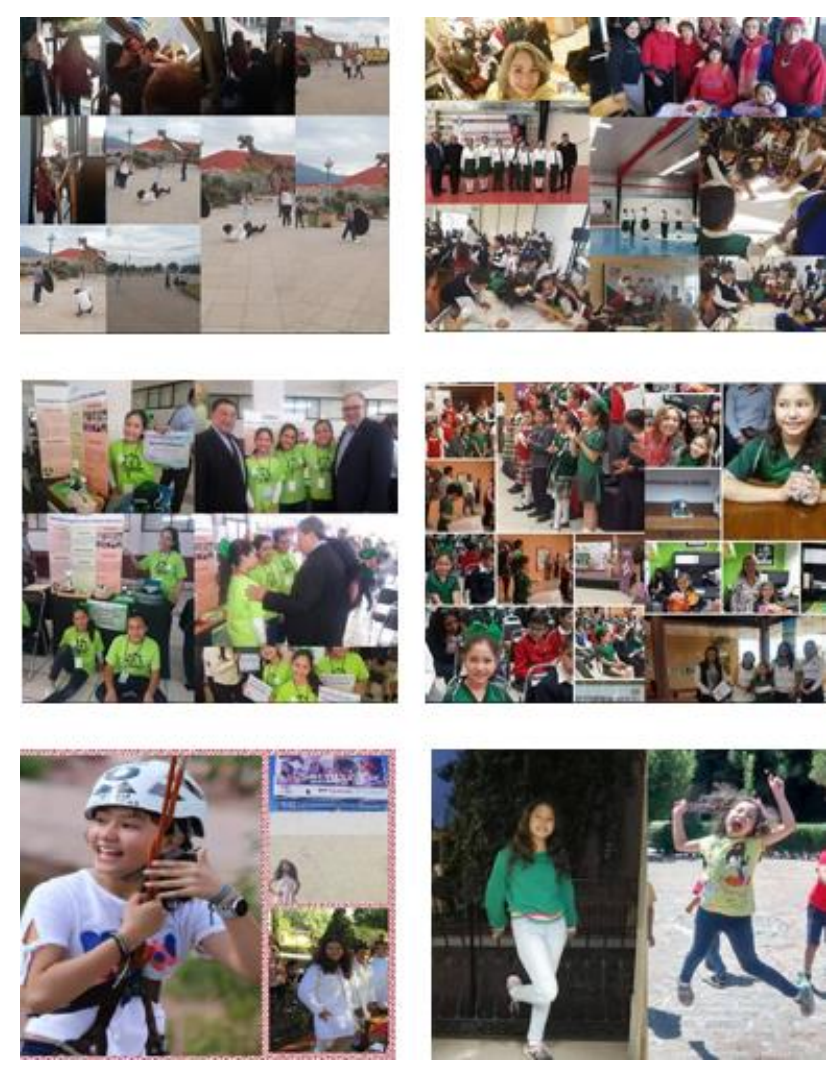

Figura 1 BILT en diferentes actividades

\section{Conclusiones}

En este estudio se concluye que el desarrollo de la Inteligencia Emocional es importante para el logro del empoderamiento. El análisis de los resultados revela que dos de las variables más importantes para el logro de lo anterior, son las relativas a la dimensión de la competencia personal, en este sentido destacan la conciencia emocional y la valoración adecuada de sí mismo. Este hallazgo es importante por cuanto se evidencia que es importante el auto conocimiento para luego pasar al desarrollo de otras capacidades que se vinculen ya con los otros.
El ser consciente de sí mismo da la posibilidad de entender las capacidades que tiene y que se pueden desarrollar. Los niños no lo pueden hacer solos, los adultos a su alrededor tienen la posibilidad de colaborar en este proceso. Las estrategias que cada uno pueda emprender tendrán que iniciar por reconocer las propias emociones, las fortalezas y debilidades, entonces, siendo los niños y adultos conscientes de lo anterior, podrán lograr mejores acciones para el logro de los objetivos de empoderamiento.

Empoderar a un niño es una actividad delicada, pues es ahí, en el proceso, dónde si no se perfila con delicadeza, con objetivos precisos y con un correcto desarrollo de la Inteligencia Emocional, podemos establecer estrategias equivocadas o en contra del mismo niño. Empoderar implica responsabilidad, sensibilidad y empatía. Los futuros líderes deben de formarse desde la niñez en un empoderamiento positivo.

\section{Referencias}

Álvarez, R. (2011). El concepto de niñez en la convención sobre los derechos del niño y en la legislación mexicana. Instituto de Investigaciones Jurídicas, UNAM. México. Recuperado de

http://ru.juridicas.unam.mx/xmlui/bitstream/han dle/123456789/11917/el-concepto-de-ninez-enla-convencion-sobre-los-derechos-del-nino-yen-la-legislacionmexicana.pdf? sequence $=5 \&$ isAllowed $=y$

Cárdenas, E. (S/F). La situación de la infancia y la adolescencia en México. Instituto de Investigaciones Jurídicas, UNAM. México. Recuperado de

https://archivos.juridicas.unam.mx/www/bjv/lib ros/9/4242/5.pdf

Enesco, I. (2008). El concepto de la infancia a lo largo de la historia. Recuperado de https://webs.ucm.es/info/psicoevo/Profes/Ileana Enesco/Desarrollo/La_infancia_en_la_historia. pdf

Extremera, N.; Fernández-Berrocal, P. (2004). El papel de la inteligencia emocional en el alumnado: evidencias empíricas. Revista Electrónica de Investigación Educativa [en línea], Vol. 6, No. 2, 2004. Recuperado en: http://redie.uabc.mx/vol6no2/contenidoextremera.html

TERRAZAS-MEDINA, Tamara Isabel, MARTÍNEZ-CÁRDENAS, Juana María, MORALES-SILVA, Rosa Elia y PÉREZ-CASTRO, Francisco Isaí. Historia de vida que empodera. Revista Transdisciplinaria de Estudios Migratorios. 2019 
Fernández, R. (2005). La inteligencia emocional como estrategia educativa inclusiva. Innovación educativa [en línea], n. ${ }^{\circ}$ 21, 2011: pp. 133-150 133.Recuperado de: file:///C:/Users/15ab111la/Downloads/29-716-1-PB\%20(1).pdf

Goleman, D. (1999). La práctica de la Inteligencia Emocional. Editorial Kairós. Barcelona, España.

Goleman, D. (1998). La Inteligencia Emocional en la práctica. Editorial Kairós. Barcelona, España.

Goleman, D. (1995). La inteligencia emocional. Editorial Kairós. Barcelona, España.

Hernández, K. S. (2009). El método historia de vida: alcances y potencialidades. Recuperado de http://www.gestiopolis.com/economia/metodode-investigacióncualitativa.htm

Lantieri, L. Goleman, D. Inteligencia emocional infantil y juvenil. Ed. Aguilar. 2009

UNICEF. (2005). La infancia amenazada. Estado mundial de la infancia. Recuperado de https://www.unicef.org/spanish/sowc05/childho oddefined.html4

UNICEF. (2017). El camino al empoderamiento de las niñas en América Latina y el Caribe: 5 derechos. Recuerdo de https://www.unicef.org/lac/media/1436/file

Vivas, M; Gallego, D; Gonzáles, B. Educar las emociones. Producciones editoriales, 2007. Mérida, Venezuela. 